\title{
Methicillin-resistant Staphylococcus aureus colonization of infectious and non-infectious skin and soft tissue lesions in patients in Tehran
}

Haniyeh Khalili', Shahin Najar-Peerayeh ${ }^{1}$, Mona Mahrooghi ${ }^{1}$, Parvin Mansouri ${ }^{2}$ and Bita Bakhshi ${ }^{* *}$

\begin{abstract}
Background: The most common clinical manifestations of Staphylococcus aureus strains in the community are skin and soft-tissue infections. S. aureus could colonize the body sites and complicate the pathogenesis of skin diseases. S. aureus colonization is a risk factor for severe conditions such as bone and joint infections, pneumonia, bacteremia, and endocarditis. This study aimed to investigate the prevalence of $S$. aureus strains in skin and soft tissue infections and other skin disorders in patients referring to dermatology clinics and to evaluate the antibiotic resistance pattern and molecular characteristics of $S$. aureus isolates.
\end{abstract}

Methods: Skin swabs were collected from the lesional sites in 234 outpatients referring to dermatology clinics in three hospitals in Tehran. Antibiotic susceptibility, biofilm formation, and hemolysis tests were performed for isolates. PCR was done for SCCmec typing, agr grouping, and virulence genes detecting.

Results: The prevalence of $S$. aureus strains among patients with skin and soft-tissue infections and other skin lesions was 44.77\% (30/67) and 44.91\% (75/167), respectively. Also, 59 (56.19\%) isolates were MRSA, 35.57\% were HA-MRSA, and $30.5 \%$ were CA-MRSA. The psma gene was more prevalent (62.8\%) among isolates, followed by hlaa (56.1\%), tsst-1 (15.2\%) eta (13.3\%), etb (6.6\%), and pvI (2.8\%). The agr specificity groups I, II, III, and IV were identified in 49.5, 21.9, 11.4, and $14.2 \%$ of S. aureus isolates, respectively. Most (56\%) S. aureus isolates produced a moderate biofilm, and $23.8 \%$ of them produced strong biofilms. a-hemolysin (46.6\%), $\beta$-hemolysin (25.7\%), $\gamma$-hemolysin (19\%), and both a and $\beta$-hemolysin (5.7\%) were also produced by isolates.

Conclusion: The present study results indicated high colonization of skin lesions by HA-MRSA and CA-MRSA clones; MRSA strains were more resistant to antibiotics, contained various toxin genes, and were able to form biofilms. Therefore, they could play a vital role in the pathogenesis of various skin diseases; also, they could spread and cause infections in other body sites. Eradication and decolonization strategies could prevent recurrent infections and the spread of resistant strains and improve skin conditions.

Keywords: HA-MRSA, CA-MRSA, Skin and soft tissue, Biofilm

*Correspondence: b.bakhshi@modares.ac.ir

1 Department of Bacteriology, Faculty of Medical Sciences, Tarbiat Modares University, Tehran, Iran

Full list of author information is available at the end of the article

\begin{abstract}
Introduction
Staphylococcus aureus is an opportunist bacterium that causes a variety of clinical infections, ranging from skin and soft-tissue and device-related infections to bacteremia and endocarditis $[1,2]$. On the other hand, S. aureus is a member of the commensal bacteria of the mucosal microbiome. Asymptomatic colonization of anterior nares by S. aureus
\end{abstract}


in healthy individuals is approximately $25-30 \%$, while the colonization of other body sites is less frequent $[3,4]$. When the skin microbiome is imbalanced, and pro-inflammatory cytokines change the skin environment, disorders such as atopic dermatitis, rosacea, and psoriasis appear. S. aureus is one of the most common bacteria found in these conditions, especially in atopic dermatitis [5].

S. aureus expresses several virulence factors that contribute to the colonization of the skin and invasion of epidermal barriers, including polysaccharide intercellular adhesin (PIA) molecular polymer, which triggers adhesion and biofilm formation. Also, S. aureus toxins, such as phenol-soluble modulins, exfoliative toxin A and $B$ (EtA, EtB), toxic shock syndrome toxin 1 (TSST1), hemolysins, and Panton-Valentine leukocidin (PVL) toxin, induce immune cell death and cytokine release [5, 6]. Additionally, methicillin-resistant S. aureus (MRSA) is a significant cause of skin and soft-tissue colonization and infections, especially community-associated MRSA (CA-MRSA), which is more virulent and grows faster than hospital associated-MRSA (HA-MRSA) [7, 8]. However, the actual role of virulence factors in the pathogenesis of different $S$. aureus clinical manifestations is unclear. A few studies have been conducted on S. aureus prevalence in skin and soft-tissue lesions in our country. Therefore, this study aimed to evaluate the prevalence of S. aureus in infectious and non-infectious skin and softtissue lesions in patients referring to dermatology clinics in Tehran and to evaluate antibiotic resistance pattern and molecular characteristics of $S$. aureus isolates.

\section{Results}

\section{Characteristics of patients and bacterial isolates}

In this study, 234 patients with skin disorders were categorized into two distinct groups based on their clinical impressions, including 67 patients with skin and soft-tissue infections (SSTIs) such as blister, impetigo, cellulitis, erysipelas, abscess, furuncles, necrotizing fasciitis, wounds, and insect/animal bites and 167 patients with non-infectious skin lesions such as eczema, erythematous skin lesions, psoriasis, drug reactions, erythema nodosum, insect/animal bites, and carcinoma. Table 1 shows the general characteristics of the patients included in this study. A total of $105 \mathrm{~S}$. aureus isolates were collected from the patients: 30 (28.57\%) isolates from SSTIs and $75(71.42 \%)$ isolates from non-infectious skin lesions. The mean age of the patients with $S$. aureus colonization of skin lesions was 22.3years (ranging from 30 days to 85 years). Also, $51.4 \%(54 / 105)$ of the patients were female (Table 1).

The prevalence of $S$. aureus strains was $44.77 \%$ (30/67) and 44.91\% (75/167) among patients with SSTIs and other skin lesions, respectively. There was no significant difference in $S$. aureus colonization of skin lesions between the two groups of patients $(p=.985, \mathrm{OR}=0.997$, 95\% CI: 0728-1.366).

\section{Antibiotic susceptibility testing}

Among $105 \mathrm{~S}$. aureus isolates tested, the highest antibiotic resistance rate was related to penicillin $(98,93.3 \%)$, followed by cefoxitin $(59,56.1 \%)$, clindamycin (48, $47.6 \%)$, erythromycin (42,37.1\%), and tetracycline (34, $32.3 \%)$. Additionally, resistance to rifampin, mupirocin, and linezolid was observed in 26 (27.6\%), 22 (20.9\%), and $17(16.1 \%)$ isolates, respectively. Fortunately, the isolates susceptibility to chloramphenicol (103, 98.1\%), gentamicin $(100,95.3 \%)$, ciprofloxacin $(99,94.3 \%)$, and amikacin (94, 89.6\%) was high (Fig.1). All the strains $(100 \%)$ isolated from SSTIs were resistant to penicillin (Fig.1).

Among 105 isolates, 59 (56.1\%) isolates were methicillin-resistant $S$. aureus (MRSA). The prevalence of

Table 1 Demographic characteristics of the two patient groups

\begin{tabular}{|c|c|c|c|c|}
\hline Characteristics & Infectious Group & Non-infectious Group & Total & $P \leq .05, \mathrm{OR} ; 95 \% \mathrm{Cl}$ \\
\hline Total patients & $67(28.63 \%)$ & 167(71.36\%) & $234(100 \%)$ & - \\
\hline Female /Male & $46 / 21$ & $73 / 94$ & $119 / 115$ & $.001,1.571(1.240-1.989)$ \\
\hline Median age years & 23.5 & 21.7 & 22.3 & - \\
\hline Infant $(\leq 2)$ & $11(16.4 \%)$ & $44(26.3 \%)$ & $55(23.5 \%)$ & $.105,0.623(0.342-1.132)$ \\
\hline Children (3-18) & $22(32.8 \%)$ & $54(32.3 \%)$ & $76(32.4 \%)$ & $.941,1.015(0.676-1.525)$ \\
\hline Adults $(18<)$ & $34(50.7 \%)$ & $69(41.3 \%)$ & $103(44 \%)$ & $.189,1.228(0.912-1.653)$ \\
\hline Patients with S. aureus & $30(44.7 \%)$ & $75(44.9 \%)$ & $105(44.8 \%)$ & $.985,0.997(0728-1.366)$ \\
\hline Female /Male & $20 / 10$ & $34 / 41$ & $54 / 51$ & $.048,1.471(1.031-2.097)$ \\
\hline Median age years & 24.2 & 22.6 & 23.4 & - \\
\hline Infant $(\leq 2)$ & $6(20 \%)$ & $20(26.6 \%)$ & $26(24.7 \%)$ & $.475,0.750(0.334-1.683)$ \\
\hline Children (3-18) & $9(30 \%)$ & $20(26.6 \%)$ & $29(37.6 \%)$ & $.730,1.125(0.580-2.183)$ \\
\hline Adults $(18<)$ & $15(50 \%)$ & $35(46.6 \%)$ & $50(47.6 \%)$ & $.757,1.071(0.696-1.650)$ \\
\hline
\end{tabular}




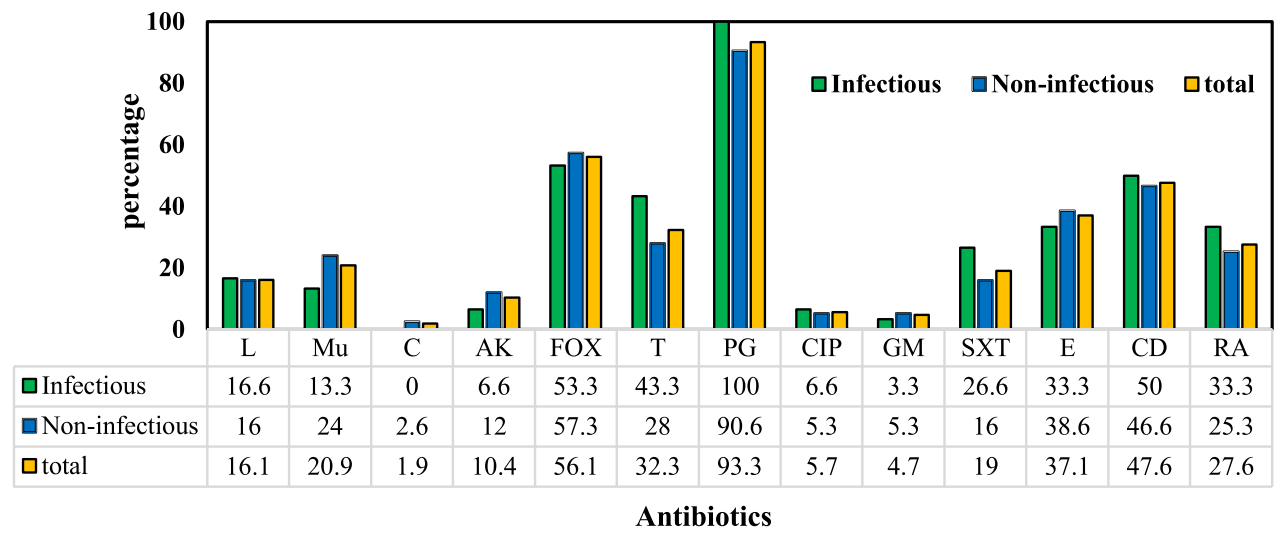

L: Linezolid, Mu: Mupirocin, C: Chloramphenicol, AK: Amikacin, FOX: Cefoxitin, T: Tetracycline, PG: Penicillin, CIP: Ciprofloxacin, GM: Gentamicin, SXT: Trimethoprim/Sulfamethoxazole, E: Erythromycin, CD: Clindamycin, RA: Rifampin

Fig. 1 Antibiotic resistance pattern of S. aureus strains isolated from infectious and non-infectious lesions. L: Linezolid, Mu: Mupirocin, C: Chloramphenicol, AK: Amikacin, FOX: Cefoxitin, T: Tetracycline, PG: Penicillin, CIP: Ciprofloxacin, GM: Gentamicin, SXT: Trimethoprim/ Sulfamethoxazole, E: Erythromycin, CD: Clindamycin, RA: Rifampin

MRSA among S. aureus strains isolated from SSTIs and other skin lesions was 53.3\% (16/30) and 57.3\% (43/75), respectively.

Penicillin resistance rate (93\%) was similar in MRSA and MSSA strains (Table 2). However, MRSA strains were more resistant to the antibiotics tested compared to MSSA isolates, including amikacin ( $16 \%$ vs. $2 \%)$, trimethoprim/sulfamethoxazole ( $28 \%$ vs. $6 \%$ ), clindamycin $(62 \%$ vs. $28 \%)$, and rifampin ( $35 \%$ vs. $17 \%)$. High resistance to clindamycin (62\%), rifampin (35\%), and linezolid was observed in MRSA strain isolated from non-infectious skin lesions (Table 2). There was no significant difference regarding the prevalence of MRSA in skin lesions between the two groups of patients $(p=.766, \mathrm{OR}=1.105,95 \% \mathrm{CI}: 0.571-2.139)$.

Most MRSA strains were simultaneously resistant to more than three antimicrobial classes. One MRSA isolate was resistant to all the antibiotics tested, except amikacin. Also, six MRSA isolates were simultaneously resistant to linezolid, mupirocin, penicillin, clindamycin, and rifampin. Additionally, two MSSA isolates were susceptible to all the antibiotics tested, and two other MSSA

Table 2 Prevalence of antibiotics resistance among MRSA and MSSA strains from the two groups of lesions

\begin{tabular}{|c|c|c|c|c|c|c|c|c|}
\hline \multicolumn{5}{|c|}{ MRSA } & \multicolumn{4}{|l|}{ MSSA } \\
\hline & $\begin{array}{l}\text { Infectious Group } \\
N=16\end{array}$ & $\begin{array}{l}\text { Non- } \\
\text { infectious } \\
\text { Group } \\
N=43\end{array}$ & $\mathrm{X}^{2}, P$-Value & $\begin{array}{l}\text { Total } \\
N=59\end{array}$ & $\begin{array}{l}\text { Infectious Group } \\
N=14\end{array}$ & $\begin{array}{l}\text { Non- } \\
\text { infectious } \\
\text { Group } \\
N=32\end{array}$ & $X^{2}, P$-Value & $\begin{array}{l}\text { Total } \\
N=46\end{array}$ \\
\hline$L$ & $3(18.7 \%)$ & $8(32 \%)$ & .990 & $11(18.6 \%)$ & $2(14.2 \%)$ & $4(12.5 \%)$ & .869 & $6(13.3 \%)$ \\
\hline $\mathrm{Mu}$ & $1(6.2 \%)$ & $12(27.9 \%)$ & .074 & $13(22 \%)$ & $3(21.4 \%)$ & 6(18.7\%) & .833 & $9(19.5 \%)$ \\
\hline C & 0 & $2(4.6 \%)$ & .278 & $2(3.3 \%)$ & 0 & 0 & - & 0 \\
\hline AK & $2(12.5 \%)$ & $8(18.6 \%)$ & .578 & $10(16.9 \%)$ & 0 & $1(3.1 \%)$ & .504 & $1(2.1 \%)$ \\
\hline $\mathrm{T}$ & $8(50 \%)$ & $13(30.2 \%)$ & .159 & $21(35.5 \%)$ & $5(35.7 \%)$ & $8(25 \%)$ & .458 & $13(28.2 \%)$ \\
\hline$P G$ & $16(100 \%)$ & $39(90.6 \%)$ & .206 & $55(93.2 \%)$ & $14(100 \%)$ & $29(90.6 \%)$ & .236 & $43(93.4 \%)$ \\
\hline CIP & $2(12.5 \%)$ & $3(6.9 \%)$ & .498 & $5(8.7 \%)$ & 0 & $1(3.1 \%)$ & .504 & $1(2.1 \%)$ \\
\hline GM & $1(6.2 \%)$ & $4(9.3 \%)$ & .708 & $5(8.7 \%)$ & 0 & 0 & - & 0 \\
\hline SXT & $7(43.7 \%)$ & $10(23.2 \%)$ & .122 & $17(28.8 \%)$ & $1(7.1 \%)$ & $2(6.2 \%)$ & .910 & $3(6.5 \%)$ \\
\hline E & $8(50 \%)$ & $16(37.2 \%)$ & .374 & $24(40.6 \%)$ & $5(35.7 \%)$ & $13(40.6 \%)$ & .754 & 18(39.1\%) \\
\hline $\mathrm{CD}$ & $11(68.7 \%)$ & $28(65.5 \%)$ & .793 & $37(62.7 \%)$ & $2(14.2 \%)$ & $9(28.1 \%)$ & .311 & $11(23.9 \%)$ \\
\hline RA & $6(37.5 \%)$ & $15(34.8 \%)$ & .852 & $21(35.5 \%)$ & $1(7.1 \%)$ & $4(12.5 \%)$ & .591 & $5(10.8 \%)$ \\
\hline
\end{tabular}

L linezolid, Mu mupirocin, C chloramphenicol, AK amikacin, FOX cefoxitin, $T$ tetracycline, $P G$ penicillin, CIP ciprofloxacin, GM gentamicin, SXT trimethoprim/ sulfamethoxazole, $E$ erythromycin, $C D$ clindamycin, $R A$ rifampin 
isolates were susceptible to all the antibiotics tested, except erythromycin.

\section{Detection of resistance genes}

MRSA strains isolated from SSTIs belonged to SCCmec II (4, 25\%), SCCmec III (3, 18.7\%), SCCmec IVa (1, $6.2 \%)$, and SCCmec IVb (3, 18.7\%), and 5 (31.2\%) isolates were untypeable. Also, SCCmec I (2, 4.6\%), SCCmec II (4, 9.3\%), SCCmec III (8, 18.6\%), SCCmec IVa (4, 9.3\%), SCCmec IVb (4, 9.3\%), SCCmec IVc (1, 2.3\%), SCCmec IVd (2, 4.6\%), and SCCmec V (3, 6.9\%) as well as 11 (25.5\%) unknown isolates were identified in the strains isolated from non-infectious skin lesions.

The int1gene was identified in $42(40 \%)$ isolates, among which 29 isolates were MRSA, and 13 isolates were MSSA.

\section{Agr grouping and detection of virulence genes}

The toxin genes detected in the isolates are shown in Table 3. The most prevalent gene among 105 isolates was psm $\alpha(66,62.8 \%)$, followed by hla $\alpha(59,56.1 \%)$, tsst- $1(16$, $15.2 \%)$, eta (14, 13.3\%), etb (7, 6.6\%), and pvl (3, 2.8\%). The $p v l$ gene was detected only in MSSA isolates, and exfoliative toxin genes were more prevalent in MSSA isolates (Table 3). The prevalence of hlaa gene was significantly higher in the strains from non-infectious skin lesions than in the strains from infectious skin lesions $\left(p=0.034 ; \chi^{2}=0.397 ; 95 \%\right.$ CI: $\left.0.167-0.946\right)$. There was no significant difference regarding the prevalence of other virulence genes between the two groups of isolates (Table 3).
The agr specificity groups I, II, III, and IV were identified in $49.5,21.9,11.4$, and $14.2 \%$ of the isolates, respectively, and 3 (2.8\%) isolates were untypeable (Table 3 ).

\section{Biofilm formation and hemolysis pattern}

Most (59/105, 56\%) S. aureus isolates produced a moderate biofilm, and $23.8 \%$ (25/105) were strong-biofilm producers (Table 3). Strong biofilm-producing isolates were more prevalent among MRSA strains (19/59; 32\%) than MSSA isolates $(6 / 46,13 \%)$.

The prevalence of strong biofilm-producing isolates in infectious and non-infectious skin lesions was $20 \%$ $(6 / 30)$ and 25\% (19/75), respectively. Among 25 strong biofilm-producing isolates, the tsst-1 gene was found in $5(20 \%)$ isolates, hla $\alpha$ in $16(64 \%)$ isolates, eta and etb in $6(24 \%)$ isolates each, and $p v l$ in 1 (4\%) isolate. Additionally, 36\% (9/25) of strong biofilm-producing isolates were CA-MRSA, 24\% (6/25) were HA-MRSA, $20 \%(5 / 25)$ were untypeable MRSA, and $20 \%(5 / 25)$ were MSSA. Biofilm formation was significantly higher in MRSA strains (Table 3).

All the isolates also produced hemolysin, including $\alpha$-hemolysin (49/105, 46.6\%), $\beta$-hemolysin (27/105, $25.7 \%), \gamma$-hemolysin $(20 / 105,19 \%)$, and both $\alpha$ and $\beta$-hemolysin (9/105, 5.7\%).

\section{Discussion}

In this study, the presence of $S$. aureus strains in various skin and soft tissue lesions was evaluated. $S$. aureus SSTIs have various clinical presentations such

Table 3 Prevalence of toxin genes, agr groups, and biofilm formation in S. aureus isolates from the two groups

\begin{tabular}{|c|c|c|c|c|c|c|c|}
\hline & $\begin{array}{l}\text { Infectious Group } \\
N=30\end{array}$ & $\begin{array}{l}\text { Non-infectious } \\
\text { Group } N=75\end{array}$ & $x^{2} P$-Value & $\begin{array}{l}\text { MRSA } \\
N=59\end{array}$ & $\begin{array}{l}\text { MSSA } \\
N=46\end{array}$ & $\begin{array}{l}X^{2} \\
P \text {-Value }\end{array}$ & $\begin{array}{l}\text { Total } \\
N=105\end{array}$ \\
\hline tsst-1 & $4(13.3 \%)$ & $12(16 \%)$ & .732 & 13(23.2\%) & $3(6.5 \%)$ & .028 & 16(15.2\%) \\
\hline $\mathrm{pvl}$ & $2(6.6 \%)$ & $1(1.33)$ & .138 & 0 & $3(6.5 \%)$ & .047 & $3(2.8 \%)$ \\
\hline Eta & $3(10 \%)$ & $11(14.6 \%)$ & .525 & $7(11.8 \%)$ & $7(15.2 \%)$ & .616 & 14(13.3\%) \\
\hline etb & $3(10 \%)$ & $5(6.6 \%)$ & .561 & $2(3.3 \%)$ & $5(10.8 \%)$ & .127 & $7(6.6 \%)$ \\
\hline hlaa & $12(40 \%)$ & $47(62.6 \%)$ & .034 & $38(64.4 \%)$ & $21(45.6 \%)$ & .055 & $59(56.1 \%)$ \\
\hline psma & $20(66.6 \%)$ & $46(61.3 \%)$ & .609 & $38(64.4 \%)$ & 28(60.8\%) & .710 & $66(62.8 \%)$ \\
\hline agr / & $11(36.6 \%)$ & $41(54.6 \%)$ & .096 & $25(42.3 \%)$ & $27(58.6 \%)$ & .097 & $52(49.5 \%)$ \\
\hline agr // & $9(30 \%)$ & $14(18.6 \%)$ & .205 & 10(16.9\%) & 13(28.2\%) & .164 & 23(21.9\%) \\
\hline agr III & $4(13.3 \%)$ & $8(10.6 \%)$ & .698 & $11(18.6 \%)$ & $1(2.1 \%)$ & .008 & 12(11.4\%) \\
\hline $\operatorname{agr} I V$ & $3(10 \%)$ & $12(16 \%)$ & .427 & 10(16.9\%) & $5(10.8 \%)$ & .377 & $15(14.2 \%)$ \\
\hline S-biofilm & $6(20 \%)$ & $19(25.3 \%)$ & .562 & 19(32.2\%) & $6(13 \%)$ & .022 & $25(23.8 \%)$ \\
\hline M-biofilm & 15(50\%) & $44(58.6 \%)$ & .419 & $24(40.6 \%)$ & $35(76 \%)$ & .000 & $59(56.1 \%)$ \\
\hline W-biofilm & $6(20 \%)$ & $9(12 \%)$ & .290 & $13(22 \%)$ & $2(4.3 \%)$ & .010 & $15(14.2 \%)$ \\
\hline No-biofilm & $3(10 \%)$ & $3(4 \%)$ & .231 & $3(5 \%)$ & $3(6.5 \%)$ & .757 & $6(5.7 \%)$ \\
\hline
\end{tabular}

$S$ strong, $M$ moderate, $W$ weak 
as blister, impetigo, cellulitis, abscess, furuncles, and necrotizing fasciitis. This bacterium is not a member of the skin and soft tissue normal microbiota; however, its colonization in the skin and soft tissues complicates many skin diseases pathogenesis or persistence [9]. In our country, a few studies have been conducted to evaluate the presence of $S$. aureus in skin and soft tissue lesions. In this study, $S$. aureus colonization was evaluated in two groups of patients with infectious and non-infectious lesions. The results showed that the rate of $S$. aureus colonization was almost the same in both groups of patients $(44.7 \%$ vs. $44.9 \%)$. In other studies in Iran, the prevalence of $S$. aureus has been reported to be 22.6 and $51.3 \%$ in SSTIs in Shiraz and Tehran, 33\% in atopic dermatitis, and $59.1 \%$ in pemphigus vulgaris legions, respectively [10-13], while the prevalence of MRSA has been reported to be 46.8 and $60 \%$ in SSTIs in Shiraz and Tehran, $42.2 \%$ in pemphigus vulgaris, and $33 \%$ in atopic dermatitis lesions [10-13]. Unexpectedly, the prevalence rate of MRSA strains was higher in non-infectious lesions (57.3\%) than in infectious lesions (53.3\%). This is likely due to underlying chronic diseases in patients in the non-infectious group, such as eczema, psoriasis, and chronic erythema nodosum.. In some studies in Geneva, Beijing, and Vancouver, the incidence rate of MRSA isolates has been reported to be 7,3 , and $54 \%$ in SSTIs, respectively [14-16]. The high prevalence rate of MRSA strains in outpatients in this study and other research in Iran could be extremely worrying due to the relatively high resistance of MRSA strains to the antibiotics tested (Table 2), especially to clindamycin (72\%), and to some extent, to mupirocin (22\%); treatment or decolonization efforts for MRSA strains seems to be problematic. Decolonization with chlorhexidine body washes or diluted sodium hypochlorite (bleach) baths is recommended [9].

Resistance to penicillin was very high, and all the strains isolated from infectious lesions were completely resistant to penicillin (Fig. 1). S. aureus strains isolated from non-infectious lesions were more resistant to the antibiotics tested, and 57\% of them were MRSA. This group of patients had various chronic diseases, and most of them had a history of hospitalization. SCCmec type I to $\mathrm{V}$ were detected in MRSA strains isolated from infectious and non-infectious lesions, indicating that both CA-MRSA and HA-MRSA are circulating in Tehran community. Some studies have shown the simultaneous presence of CA-MRSA and HA-MRSA in the community and hospital settings [17-19]; therefore, the use of SCCmec type as a marker to differentiate between CA-MRSA and HA-MRSA may not be useful.

Regarding virulence genes, the $p v l$ gene was detected only in three strains, all of which were MSSA; two strains were isolated from four- and six-month-old newborns, and one strain was isolated from eczema lesions of a 65-year-old man. PVL has been reported to be associated with MRSA strains, especially CAMRSA [15]. However, recent research has shown that PVL is found in both methicillin-sensitive and -resistant strains in the community and healthcare settings, and its incidence rate depends on the sample size and the geographical regions [20-23]. Therefore, the PVLbacteriophage acquisition event is probably independent of the mecA gene acquisition.

The presence of toxin genes, such as psma, hla $\alpha$, tsst1 , eta, and etb, was also evaluated in this study. Bacterial toxins may impair the integrity of the skin's defense barriers, allowing bacteria and antigens to penetrate in the sub-epithelium. Also, the toxic shock syndrome toxin-1 (TSST-1) is a strong super-antigen that induces an inflammatory response by activating the immune system [24-26]. Generally, $15 \%$ of $S$. aureus isolates carried the tsst-1 gene; also, the psm $\alpha$ and hla $\alpha$ genes were present in more than 55\% of the isolates. Exfoliative toxin genes were identified in $20 \%$ of the isolates, and $\alpha, \beta$, and $\gamma$ cytotoxins were produced by the isolates. All of these cytotoxins are likely to play a role in the structural and immune condition of patients' skin.

Biofilm formation is associated with many $S$. aureus infections. Biofilm formation protects microorganisms against natural skin antimicrobials, immune responses, environmental stresses, and antibiotic treatment [2729]. In general, $80 \%$ of the isolates in this study produced strong or moderate biofilm, and no statistical differences were found in biofilm formation between the strains isolated from the two groups of patients. However, biofilm formation was significantly higher in MRSA strains than in MSSA (Table 3). Biofilm formation is a typical feature of $S$. aureus isolates from various clinical specimens [27-30]. After the maturation of biofilm, S. aureus isolates could disseminate and colonize new regions. Therefore, biofilm formation is necessary for colonization and infection.

Evaluation of agr specificity groups detected in this study showed that the frequency of agr specificity group I (49.5\%) was higher than other agr groups among the isolates. Agr group I is dominant in most $S$. aureus isolates from various clinical specimens [31, 32]. The prevalence of $a g r$ specificity group III was significantly $(p=.008)$ higher in MRSA strains than in MSSA; further studies are needed to confirm the association between MRSA strains and $a g r$ specificity group III in skin and soft tissue lesions.

Although in this study, the relationship between $S$. aureus isolates and the type of skin lesions was not evaluated by genotypic analysis such as MLST, PFGE, 
or other typing methods, but high clonal diversity has been reported by researchers among $S$. aureus isolates from various skin and soft tissue lesions. For example, Zhao et al. (2012) [15] suggested similar epidemiology for community-acquired and hospital-acquired $S$. aureus infections. Yeung et al. (2011) [33] concluded there was no predominant clonal type among $S$. aureus isolates from atopic dermatitis lesions. However, SCCmec typing, agr grouping, and virulence typing results in the present study indicated no significant difference between the isolates from various skin lesions, except for hla gene that was significantly more prevalent in non-infection legions. Further studies are needed to investigate the relationship between isolates and different skin legions in our country.

\section{Conclusion}

In this study, the prevalence and molecular characteristics of MSSA and MRSA strains isolated from infectious and non-infectious skin and soft-lesions were investigated in Iranian dermatology clinics. The results indicated high colonization of skin lesions by HA-MRSA and CA-MRSA clones; MRSA strains were more resistant to antibiotics commonly used in the treatment of S.aureus infection. Furthermore, these isolates contained various toxin genes and were able to form biofilm, which are considered as essential factors for generating antibioticresistant infections. S. aureus could play a major role in the pathogenesis of various skin diseases; also, it could spread and cause infections in other body sites. The present study results showed a high prevalence rate of MRSA in outpatients, given that in the past, there was an emphasis on recognizing these isolates in skin and softtissue lesions and determining their antibiogram profile. Eradication and decolonization strategies could prevent recurrent infections and the spread of resistant strains and improve skin conditions.

\section{Material \&Methods}

\section{Characteristics of patient and bacterial isolates}

Skin swabs were collected from the lesional sites in outpatients by dermatologists in three hospitals (two general and one pediatric hospitals) in Tehran during April 2016-2017. Demographic and clinical data of patients were recorded. Patients who took antibiotics 2 weeks prior to sampling were excluded. The collected swab samples were transported promptly in thioglycolate broth to the clinical microbiology laboratory. After overnight incubation at $37^{\circ} \mathrm{C}$, the samples were cultured on blood agar medium at $37^{\circ} \mathrm{C}$. S. aureus strains was identified using the following tests, including gram staining, catalase test, mannitol fermentation, slide and tube coagulase tests, DNase production, and presence of the nuc gene [34].

\section{Antibiotic susceptibility testing}

Antimicrobial susceptibility test was performed for the isolates according to the Clinical and Laboratory Standards Institute [35]. Antibiotic disks used were as follows; clindamycin $(2 \mu \mathrm{g})$, mupirocin $(200 \mu \mathrm{g})$, chloramphenicol $(30 \mu \mathrm{g})$, amikacin $(30 \mu \mathrm{g})$, penicillin $(10 \mu \mathrm{g})$, gentamicin $(10 \mu \mathrm{g})$, tetracycline $(30 \mu \mathrm{g})$, ciprofloxacin $(5 \mu \mathrm{g})$, trimethoprim-sulfamethoxazole $(1.25 / 23.75 \mu \mathrm{g})$, cefoxitin $(30 \mu \mathrm{g})$, rifampin $(5 \mu \mathrm{g})$, erythromycin $(15 \mu \mathrm{g})$, and linezolid $(30 \mu \mathrm{g})$ (MAST UK). S. aureus ATCC25923 was used as a quality control.

\section{Detection of resistance genes}

The $m e c \mathrm{~A}$ and class I integron genes were detected among the isolates by PCR. MRSA strains were typed for SCCmec. The specific primers and thermal profiles employed for PCR detection of these genes are shown in Table 4.

\section{Agr grouping and detection of virulence genes}

The presence of $p v l$, eta, etb, tsst-1, hla- $\alpha$, and psma genes was detected among the isolates by polymerase chain reaction (PCR). agr grouping was performed for all the isolates. The specific primers and thermal profiles employed for PCR detection of these genes are shown in Table 4.

\section{Biofilm formation and hemolysis pattern}

The biofilm formation ability of all the isolates was evaluated using the microtiter plate method [34]. To do so, the tryptic soy broth (TSB) supplemented with $1 \%(\mathrm{w} / \mathrm{v})$ glucose containing $107 \mathrm{CFU} / \mathrm{mL}$ of each isolate was distributed into 96 -well polystyrene microtiter plates and incubated at $37^{\circ} \mathrm{C}$ for $24 \mathrm{~h}$ while shaking at $120 \mathrm{rpm}$. After twice washing and then air-drying, a $0.1 \%$ crystal violet solution was added and after $45 \mathrm{~min}$ washed three times, and then the ethanol-acetone solution was added. A microplate reader measured the absorbance of each well at OD570 $\mathrm{nm}$ after $45 \mathrm{~min}$. $S$. aureus ATCC 35556 and S. epidermidis ATCC 12228 were used as a positive and negative control, respectively. The tests were done in duplicate at two independent times.

S. aureus hemolysis patterns were characterized in the blood agar medium containing 5\% washed rabbit red blood cells. All tests were done at two independent times. 
Table 4 Primers used in this study

\begin{tabular}{|c|c|c|c|}
\hline Primer & Sequence $\left(5^{\prime} \rightarrow 3^{\prime}\right)$ & Size (bp) & Ref. \\
\hline mecA & $\begin{array}{l}\text { F: GTGAAGATATACCAAGTGATT } \\
\text { R: ATGCGCTATAGATTGAAAGGA }\end{array}$ & 146 & [36] \\
\hline sccmec I & $\begin{array}{l}\text { F: GCTTTAAAGAGTGTCGTTACAGG } \\
\text { R: GTTCTCTCATAGTATGACGTCC }\end{array}$ & 613 & \\
\hline SCCmec II & $\begin{array}{l}\text { F: CGTTGAAGATGATGAAGCG } \\
\text { R: CGAAATCAATGGTTAATGGACC }\end{array}$ & 398 & \\
\hline sccmec III & $\begin{array}{l}\text { F: CCATATTGTGTACGATGCG } \\
\text { R: CCTTAGTTGTCGTAACAGATCG }\end{array}$ & 280 & \\
\hline sccmec Iva & $\begin{array}{l}\text { F: GCCTTATTCGAAGAAACCG } \\
\text { R: CTACTCTTCTGAAAAGCGTCG }\end{array}$ & 776 & \\
\hline scCmec IVb & $\begin{array}{l}\text { F:TCTGGAATTACTTCAGCTGC } \\
\text { R: AAACAATATTGCTCTCCCTC }\end{array}$ & 493 & \\
\hline sccmec IVc & $\begin{array}{l}\text { F: ACAATATTTGTATTATCGGAGAGC } \\
\text { R:TTGGTATGAGGTATTGCTGG }\end{array}$ & 200 & \\
\hline sccmec IVd & $\begin{array}{l}\text { F: CTCAAAATACGGACCCCAATACA } \\
\text { R:TGCTCCAGTAATTGCTAAAG }\end{array}$ & 881 & \\
\hline SCCmec V & $\begin{array}{l}\text { F: GAACATTGTTACTTAAATGAGCG } \\
\text { R:TGAAAGTTGTACCCTTGACACC }\end{array}$ & 325 & \\
\hline panF & F: ATGCACATGGTGCACATGC & - & [37] \\
\hline $\operatorname{agr} \mid-R$ & R: GTCACAAGTACTATAAGCTGCGAT & 440 & \\
\hline agr $\|-R$ & R: GTATTACTAATTGAAAAGTGCCATAGC & 572 & \\
\hline agr III-R & R: CTGTTGAAAAAGTCAACTAAAAGCTC & 406 & \\
\hline $\operatorname{agr} I V-R$ & R: CGATAATGCCGTAATAC CCG & 588 & \\
\hline tsst-1 & $\begin{array}{l}\text { F: TTATCGTAAGCCCTTTGTTG } \\
\text { R:TAAAGGTAGTTCTATTGGAGTAGG }\end{array}$ & 398 & [38] \\
\hline hla-a & $\begin{array}{l}\text { F: CGGTACTACAGATATTGGAAGC } \\
\text { R:TGGTAATCATCACGAACTCG }\end{array}$ & 744 & [38] \\
\hline psm-a & $\begin{array}{l}\text { F: TATCAAAAGCTTAATCGAACAATTC } \\
\text { R: CCCCTTCAAATAAGATGTTCATATC }\end{array}$ & 176 & [39] \\
\hline etA & $\begin{array}{l}\text { F: CTAGTG CATTTGTTATTCAAGACG } \\
\text { R:TGCATTGACACCATAGTACTTATTC }\end{array}$ & 119 & [40] \\
\hline$e t B$ & $\begin{array}{l}\text { F: ACGGCTATATACATTCAATTAATG } \\
\text { R: AAAGTTATTCATTTAATGCACTGTCTC }\end{array}$ & 200 & [40] \\
\hline Int1 & $\begin{array}{l}\text { F: CCTCCCGCACGATGATC } \\
\text { R:TCCACGCATCGTCAGGC }\end{array}$ & 188 & [41] \\
\hline
\end{tabular}

\section{Statistical analysis}

SPSS software Version 23 (SPSS Inc., Chicago, IL, USA) was used for statistical analysis. Differences in proportions were evaluated by employing the Chi-square test. Univariate analysis was performed by logistic regression. A $p$-value $<.05$ was considered statistically significant.

\footnotetext{
Abbreviations

S. aureus: Staphylococcus aureus; EtA: Exfoliative toxin A; EtB: Exfoliative toxin B; TSST-1: Toxic shock syndrome toxin 1; PVL: Panton valentine toxin; MRSA: Methicillin-resistant Staphylococcus aureus; HA-MRSA: Health care associated or hospital- associated MRSA; CA-MRSA: Community-associated MRSA; SCCmec: Staphylococcal cassette chromosome mec.
}

\section{Acknowledgments}

This work was supported by the grants (ID; 52D/1554) provided by the faculty of Medical Sciences, Tarbiat Modares University, Tehran, Iran and the Iran National Science Foundation (ID; 94006593), Tehran, Iran.

\section{Authors' contributions}

SN was responsible for the study design, data analysis, and the manuscript reviewing and editing. KH performed microbiological and molecular studies and drafted the manuscript. BB and MP advised the project. All authors approved the final version of the manuscript.

Funding

No financial support received for this research.

\section{Availability of data and materials}

The authors confirm that the data supporting the findings of this study are available within the article.

\section{Declarations}

\section{Ethics approval and consent to participate}

The study protocol was conducted according to the recommendations of the Ethics Committee of Tarbiat Modares University (Tehran, Iran) in compliance with the Ethical principles of the Declaration of Helsinki on medical research involving human subjects. This study was approved by the Ethics Committee of Tarbiat Modares University (Tehran, Iran) with ethical approval reference number IR.TMU.REC.1395.341. Informed written consent was taken from each participating patient or their parent prior to the study.

\section{Consent for publication}

Not Applicable.

\section{Competing interests}

The authors declare that they have no competing interests.

\section{Author details}

${ }^{1}$ Department of Bacteriology, Faculty of Medical Sciences, Tarbiat Modares University, Tehran, Iran. ${ }^{2}$ Department of research, Skin and Stem Cell Research Center, Tehran University of Medical Sciences, Tehran, Iran.

Received: 24 October 2020 Accepted: 6 October 2021

Published online: 18 October 2021

\section{References}

1. Balasubramanian D, Harper L, Shopsin B, Torres VJ. Staphylococcus aureus pathogenesis in diverse host environments. Pathogens and Dis 2017; 75:ftx005.

2. Ghasemian A, Peerayeh SN, Bakhshi B, Mirzaee M. Detection of accessory gene regulator groups genes and cassette chromosome mec types among Staphylococcus aureus isolated from intensive care unit patients. Asian Pac J Trop Dis. 2015;5:153-7.

3. Kluytmans J, Wertheim H. Nasal carriage of Staphylococcus aureus and prevention of nosocomial infections. Infection. 2005;33:3-8.

4. Yang E, Tan J, Eells S, Rieg G, Tagudar G, Miller L. Body site colonization in patients with community-associated methicillin-resistant and other types of S. aureus skin infections. Clin Microbiol Infect. 2010;16:425-31.

5. Geoghegan JA, Irvine AD, Foster TJ. Staphylococcus aureus and atopic dermatitis: a complex and evolving relationship. Trends in microbiol. 2018:26:484-97.

6. Tajik S, Najar-Peerayeh S, Bakhshi B, Golmohammadi R. Molecular characterization of community-associated methicillin-resistant Staphylococcus aureus in Iranian burn patients. Iran J Pathol. 2019;14:284.

7. Hiramatsu K, Ito T, Tsubakishita S, Sasaki T, Takeuchi F, Morimoto Y, et al. Genomic basis for methicillin resistance in Staphylococcus aureus. Infect Chemother. 2013;45:117-36.

8. Okuma K, Iwakawa K, Turnidge JD, Grubb WB, Bell JM, O'Brien FG, et al. Dissemination of new methicillin-resistant Staphylococcus aureus clones in the community. J Clin Microbiol. 2002:40:4289-94.

9. Kuraitis D, Williams L. Decolonization of Staphylococcus aureus in healthcare: a dermatology perspective. J Healthc Eng. 2018:53:1-8. 
10. Mehrshad S, Haghkhah M, Aghaei S. Epidemiology and molecular characteristics of methicillin-resistant Staphylococcus aureus from skin and soft tissue infections in shiraz, Iran. Turk J Med Sci. 2017;47:180-7.

11. Fagheei-Aghmiyuni Z, Khorshidi A, Soori T, Moniri R, Mousavi G. Antibiotic susceptibility pattern and the prevalence of Staphylococcus aureus isolated from skin and soft tissue in Tehran Razi skin hospital (2014-15). Feyz. 2017;21:188-96.

12. Fagheei Aghmiyuni Z, Khorshidi A, Moniri R, Soori T, SGA M. The prevalence of S. aureus skin and soft tissue infections in patients with pemphigus. Autoimmune Dis. 2016:2016.

13. Rezaei M, Chavoshzadeh Z, Haroni N, Armin S, Navidinia M, Mansouri $M$, et al. Colonization with methicillin resistant and methicillin sensitive staphylococcus aureus subtypes in patients with atopic dermatitis and its relationship with severity of eczema. Arch Pediatr Infect Dis. 2013;1(2):53-6.

14. Bouvet C, Gjoni S, Zenelaj B, Lipsky BA, Hakko E, Uçkay I. Staphylococcus aureus soft tissue infection may increase the risk of subsequent staphylococcal soft tissue infections. Int J Infect Dis. 2017;60:44-8.

15. Zhao C, Liu Y, Zhao M, Liu Y, Yu Y, Chen H, et al. Characterization of community acquired Staphylococcus aureus associated with skin and soft tissue infection in Beijing: high prevalence of PVL+ ST398. PLoS One. 2012;7:e38577.

16. Stenstrom R, Grafstein E, Romney M, Fahimi J, Harris D, Hunte G, et al. Prevalence of and risk factors for methicillin-resistant Staphylococcus aureus skin and soft tissue infection in a Canadian emergency department. CJEM. 2009;11:430-8

17. Tajik S, Najar-Peerayeh S, Bakhshi B. Hospital clones of Panton-valentine leukocidin-positive and methicillin-resistant Staphylococcus aureus circulating in the Tehran community. J Glob Antimicrob Resist. 2020;22:177-81.

18. Peng H, Liu D, Ma Y, Gao W. Comparison of community-and healthcareassociated methicillin-resistant Staphylococcus aureus isolates at a Chinese tertiary hospital, 2012-2017. Sci Rep. 2018;8:1-8.

19. Kateete DP, Bwanga F, Seni J, Mayanja R, Kigozi E, Mujuni B, et al. CA-MRSA and HA-MRSA coexist in community and hospital settings in Uganda. Antimicrob Resist Infect Control. 2019;8:94.

20. Ayepola OO, Olasupo NA, Egwari LO, Schaumburg F. Characterization of Panton-Valentine leukocidin-positive Staphylococcus aureus from skin and soft tissue infections and wounds in Nigeria: a cross-sectional study. F1000Research 2018; 7

21. Hanitsch LG, Krüger R, Hoppe P-A, Humme D, Pokrywka A, Niebank $M$, et al. Outpatient decolonization after recurrent skin infection with Panton-valentine leukocidin (PVL)-producing S. aureus - the importance of treatment repetition. PLoS One. 2020;15:e0231772.

22. Jaton L, Pillonel T, Jaton K, Dory E, Prod'hom G, Blanc D, et al. Common skin infection due to Panton-Valentine leucocidin-producing Staphylococcus aureus strains in asylum seekers from Eritrea: a genome-based investigation of a suspected outbreak. Clin Microbiol Infect. 2016;22:739 e5-. e8.

23. Khosravi AD, Hoveizavi H, Farshadzadeh Z. The prevalence of genes encoding leukocidins in Staphylococcus aureus strains resistant and sensitive to methicillin isolated from burn patients in Taleghani hospital, Ahvaz, Iran. Burns. 2012;38:247-51

24. Brauweiler AM, Goleva E, Leung DY. Th2 cytokines increase Staphylococcus aureus alpha toxin-induced keratinocyte death through the signal transducer and activator of transcription 6 (STAT6). J Invest Dermatol. 2014;134:2114-21.

25. Liu H, Archer NK, Dillen CA, Wang Y, Ashbaugh AG, Ortines RV, et al. Staphylococcus aureus epicutaneous exposure drives skin inflammation via IL-36-mediated T cell responses. Cell Host \& Microbe. 2017;22:653-66 e5.

26. Tuffs SW, Haeryfar S, McCormick JK. Manipulation of innate and adaptive immunity by staphylococcal superantigens. Pathogens. 2018;7:53
27. Mirzaee M, Najar-Peerayeh S, Behmanesh M, Moghadam MF. Relationship between adhesin genes and biofilm formation in vancomycinintermediate Staphylococcus aureus clinical isolates. Current Microbiol. 2015;70:665-70.

28. Otto M. Staphylococcal biofilms. Microbiol Spectr. 2018; 6(4): https://doi. org/10.1128/microbiolspec.GPP3-0023-2018.

29. Olia AHG, Ghahremani M, Ahmadi A, Sharifi Y. Comparison of biofilm production and virulence gene distribution among community-and hospital-acquired Staphylococcus aureus isolates from northwestern Iran. Infection, Infect Genet Evol 2020; 81:104262.

30. Ghasemian A, Najar-Peerayeh S, Bakhshi B, Mirzaee M. Comparison of biofilm formation between methicillin-resistant and methicillin-susceptible isolates of Staphylococcus aureus. Iran Biomed J. 2016;20:175.

31. Azimian A, Najar-Pirayeh S, Mirab-Samiee S, Naderi M. Occurrence of methicillin resistant Staphylococcus aureus (MRSA) among clinical samples in Tehran-Iran and its correlation with polymorphism of specific accessory gene regulator (AGR) groups. Braz J Microbiol. 2012;43:779-85.

32. Matias C, Serrano I, Van-Harten S, Mottola C, Mendes JJ, Tavares L, et al. Polymicrobial interactions influence the agr copy number in Staphylococcus aureus isolates from diabetic foot ulcers. Antonie Van Leeuwenhoek. 2018;111:2225-32.

33. Yeung M, Balma-Mena A, Shear N, Simor A, Pope E, Walsh S, et al. Identification of major clonal complexes and toxin producing strains among Staphylococcus aureus associated with atopic dermatitis. Microbes Infect. 2011;13(2):189-97.

34. Bhutia KO, Singh TS, Biswas S, Adhikari L. Evaluation of phenotypic with genotypic methods for species identification and detection of methicillin resistant in Staphylococcus aureus. Int J Applied Basic Med Res. 2012;2(2):84.

35. Clinical and Laboratory Standards Institute. Performance standards for antimicrobial susceptibility testing of anaerobic bacteria: informational supplement. 30th ed. Wayne, PA: Clinical and Laboratory Standards Institute; 2018

36. Zhang K, McClure J-A, Elsayed S, Louie T, Conly JM. Novel multiplex PCR assay for characterization and concomitant subtyping of staphylococcal cassette chromosome mec types I to V in methicillin-resistant Staphylococcus aureus. J Clin Microbiol. 2005;43:5026-33.

37. Shopsin B, Mathema B, Alcabes P, Said-Salim B, Lina G, Matsuka A, et al. Prevalence of agr specificity groups among Staphylococcus aureus strains colonizing children and their guardians. J Clin Microbiol. 2003;41:456-9.

38. Havaei SA, Azimian A, Fazeli H, Naderi M, Ghazvini K, Samiee SM, et al. Genetic characterization of methicillin resistant and sensitive, vancomycin intermediate Staphylococcus aureus strains isolated from different Iranian hospitals. ISRN Microbiol. 2012;215275.

39. Berube BJ, Sampedro GR, Otto M, Wardenburg JB. The psma locus regulates production of Staphylococcus aureus alpha-toxin during infection. Infect Immun. 2014;82:3350-8.

40. Zhao S, White DG, Ge B, Ayers S, Friedman S, English L, et al. Identification and characterization of integron-mediated antibiotic resistance among Shiga toxin-producing Escherichia coli isolates. Appl Environ Microbiol. 2001;67:1558-64

41. Stepanović S, Vuković D, Hola V, Bonaventura GD, Djukić S, Ćirković I, et al. Quantification of biofilm in microtiter plates: overview of testing conditions and practical recommendations for assessment of biofilm production by staphylococci. Apmis. 2007;115:891-9.

\section{Publisher's Note}

Springer Nature remains neutral with regard to jurisdictional claims in published maps and institutional affiliations. 